\title{
Blowup time of solutions for a small diffusive parabolic problem with exponential source
}

\section{Liping Zhu*}

\section{"Correspondence:} 78184385@qq.com College of Science, Xi'an University of Architecture and Technology,

Xi'an, 710054, China

\begin{abstract}
In this paper, we study the asymptotic behavior of blowup time for a small diffusive parabolic equation with exponential source. We prove that the blowup time of the solution converges to that of the corresponding ODE as the small diffusive approaches zero. Moreover, we show a more accurate estimate of the blowup time. Precisely, when the initial data decay near the maximum, we obtain the lower and upper bound estimates of the blowup time with a higher-order term of the peak of initial data.
\end{abstract}

MSC: 35B40; 35K20; 35K55

Keywords: blowup time; small diffusion; exponential source

\section{Introduction}

In this paper, we study the following semilinear parabolic equation:

$$
\begin{cases}u_{t}=\varepsilon \Delta u+e^{u}, & (x, t) \in \Omega \times(0,+\infty), \\ u(x, t)=0, & (x, t) \in \partial \Omega \times(0,+\infty), \\ u(x, 0)=\varphi(x), & x \in \Omega,\end{cases}
$$

where $\varepsilon>0$ is a small parameter, $\Omega$ is a bounded smooth domain in $\mathbb{R}^{N}$, and $\varphi(x)$ is a continuous (nonnegative or sign-changing) function on $\bar{\Omega}$. Equation (1.1) describes combustion processes in a medium with thermal reactions; we refer to [1] for extensive references. We know from [2] that a solution of (1.1) may blow up in finite time, that is, the maximum norm $\|u(\cdot, t)\|_{\infty}$ may diverge to $\infty$ in finite time. The maximal existence time of a solution $u$ of (1.1) in the classical sense is called the blowup time (when it is finite). We denote by $T(\varepsilon)$ the blowup time depending on the small parameter $\varepsilon$.

We first consider the corresponding ODE case. Suppose that $z(t ; \theta)$ is a solution of the equation

$$
\frac{d z}{d t}=e^{z}, \quad z(0 ; \theta)=\theta>0 .
$$

Clearly, $z(t ; \theta)$ is solved as

$$
z(t ; \theta)=-\ln \left(e^{-\theta}-t\right)
$$

(c) 2016 Zhu. This article is distributed under the terms of the Creative Commons Attribution 4.0 International License (http://creativecommons.org/licenses/by/4.0/), which permits unrestricted use, distribution, and reproduction in any medium, provided you give appropriate credit to the original author(s) and the source, provide a link to the Creative Commons license, and indicate if changes were made. 
We easily find that $z(t ; \theta) \rightarrow \infty$ as $t \rightarrow e^{-\theta}$. So it is interesting to study the asymptotic behavior of blowup time of (1.1) when $\varepsilon$ is small.

In fact, for positive initial data $\varphi(x)$, Friedman and Lacey [2] had studied the more general equation $u_{t}=\varepsilon \Delta u+f(u)$ and showed that if $f(s)=e^{s}$, then $T(\varepsilon) \rightarrow e^{-\|\varphi\|_{\infty}}$ as $\varepsilon \rightarrow 0$. We also refer to [3-5] for the estimates of blowup time for positive solutions. Later, Mizoguchi and Yanagida [6] considered the Dirichlet problem

$$
\begin{cases}u_{t}=\varepsilon \Delta u+|u|^{p-1} u, & (x, t) \in \Omega \times(0,+\infty) \\ u(x, t)=0, & (x, t) \in \partial \Omega \times(0,+\infty) \\ u(x, 0)=\varphi(x), & x \in \Omega\end{cases}
$$

where $p>1, \Omega$ is a bounded domain in $\mathbb{R}^{N}$, and $\varphi$ satisfies

$$
-\min _{x \in \bar{\Omega}} \varphi(x)<\max _{x \in \bar{\Omega}} \varphi(x) .
$$

They showed that the blowup time $T(\varepsilon)$ of the solution of this problem satisfies $T(\varepsilon) \rightarrow$ $\frac{1}{p-1}\|\varphi\|_{\infty}^{1-p}$ as $\varepsilon \rightarrow 0$. Moreover, when the initial value admits a Taylor expansion, there is a more accurate estimate of $T(\varepsilon)$. Moreover, Mizoguchi and Yanagida [7] extended this problem to the whole space and obtained similar results for the blowup time of (signchanging) solutions for the following Cauchy problem:

$$
\begin{cases}v_{t}=\Delta v+|v|^{p-1} v, & (x, t) \in \mathbb{R}^{N} \times(0, \infty), \\ v(x, 0)=\lambda \varphi(x), & x \in \mathbb{R}^{N},\end{cases}
$$

where $\lambda>0$ is a large parameter. In order to overcome the difficulty of sign-changing solutions, Mizoguchi and Yanagida used an energy functional technique introduced by Giga and Kohn [8].

Recently, Payne and Schaefer $[9,10]$ studied the blowup phenomena and derived the upper and lower bounds of blowup time for some semilinear equations under certain assumptions on reactions and initial boundary data. Also, many authors considered the rate estimates of the blowup or quenching solutions and also quenching time estimates; we refer to [11-16] and the references therein.

Remark 1.1 We note that, under the assumption that the initial data are positive, Sato [5] extended the results of Mizoguchi and Yanagida [6, 7] for power-type nonlinearity to a more general nonlinear heat equation that includes exponential-type nonlinearity. However, here we study the more general initial data that satisfy (1.4) and are sign-changing.

In the present paper, we study problem (1.1) with exponential source, which can be viewed as the limit case of (1.3) as $p \rightarrow \infty$. Also, since equation (1.1) is not scaling invariant and the initial data are sign-changing, our arguments here are more complicated. Since the energy method can be applied only to star-shaped domains, we derive here some crucial estimates of solutions for the case of small diffusion equations and extend the adapted method of comparison to sign-changing solutions on any bounded domains.

The first result is the following estimate of (1.1). 
Theorem 1.1 If $\varphi$ satisfies (1.4) and $\varepsilon>0$ is small enough, then the solution of (1.1) blows up in finite time. Furthermore, we have

$$
T(\varepsilon) \rightarrow e^{-\|\varphi\|_{\infty}}, \quad \varepsilon \rightarrow 0 .
$$

The second result is a more accurate estimate of blowup time for (1.1).

Theorem 1.2 If $\varphi$ satisfies (1.4), $\varphi\left(x_{0}\right)=\max _{x \in \bar{\Omega}} \varphi(x), \Delta \varphi\left(x_{0}\right)<0$, and $\varepsilon>0$ is small enough, then we have

$$
e^{-\varphi\left(x_{0}\right)}+\frac{1}{4} e^{-2 \varphi\left(x_{0}\right)}\left|\Delta \varphi\left(x_{0}\right)\right| \varepsilon+\circ(\varepsilon) \leq T(\varepsilon) \leq e^{-\varphi\left(x_{0}\right)}+e^{-2 \varphi\left(x_{0}\right)}\left|\Delta \varphi\left(x_{0}\right)\right| \varepsilon+\circ(\varepsilon) .
$$

Finally, we give an estimate of blowup time when the initial value $\varphi(x)$ satisfies $\varphi(x) \sim$ $\varphi\left(x_{0}\right)-c_{0}\left|x-x_{0}\right|^{2 k}$ with $c_{0}, k>0$.

Theorem 1.3 If $\varphi$ satisfies (1.4), $\varphi\left(x_{0}\right)=\max _{x \in \bar{\Omega}} \varphi(x)$, more precisely, $\varphi(x) \sim \varphi\left(x_{0}\right)-c_{0} \mid x-$ $\left.x_{0}\right|^{2 k}$ for constants $c_{0}, k>0, k \neq 1$, and $\varepsilon>0$ is small enough, then we have

$$
e^{-\varphi\left(x_{0}\right)}+c_{0} \zeta_{k} \gamma_{k} e^{-(k+1) \varphi\left(x_{0}\right)} \varepsilon^{k}+\circ\left(\varepsilon^{k}\right) \leq T(\varepsilon) \leq e^{-\varphi\left(x_{0}\right)}+c_{0} \gamma_{k} e^{-(k+1) \varphi\left(x_{0}\right)} \varepsilon^{k}+\circ\left(\varepsilon^{k}\right),
$$

where $\zeta_{k}=k^{k} /(k+1)^{k+1}$, and $\gamma_{k}$ satisfies

$$
\int_{\Omega} \frac{e^{-\frac{\left|y-x_{0}\right|^{2}}{4 \tau}}}{(4 \pi \tau)^{N / 2}}\left|y-x_{0}\right|^{2 k} d y=\gamma_{k} \tau^{k}+o\left(\tau^{k}\right) \quad \text { as } \tau \rightarrow 0 .
$$

We organize this paper as follows: In Section 2, a preliminary estimate of solutions is given. Sections 3-5 are devoted to proofs of Theorems 1.1-1.3, respectively. Finally, we give some notes for the negative initial data in Section 6.

\section{Preliminary estimates}

First, we show a preliminary lemma. Throughout this paper, letting $m:=\min _{x \in \bar{\Omega}} \varphi(x)$ and $M:=\max _{x \in \bar{\Omega}} \varphi(x)$, we denote by $T_{m}$ and $T_{M}$ the blowup times of solutions of (1.2) with $\theta=m$ and $\theta=M$, respectively.

Lemma 2.1 Suppose that (1.4) is satisfied and $\varphi(a)=M$. Then, for any $\tau>0$, there exist $\alpha$, $\varepsilon_{1}>0$ such that if $0<\varepsilon \leq \varepsilon_{1}$, then the solution of (1.1) satisfies

$$
u(x, t)>0 \quad \text { in } B_{a, \alpha} \subset \Omega
$$

for $t \in(0, \min \{\tau, T(\varepsilon)\})$, where $B_{a, \alpha}$ is the ball with center $a$ and radius $\alpha$.

Proof The solution $u$ of (1.1) can be written as

$$
u(x, t)=\int_{\Omega} \varphi(y) G(x, y ; t) d y+\int_{0}^{t} \int_{\Omega} e^{u(y, s)} G(x, y ; t-s) d y d s=I_{1}+I_{2},
$$


where $G(x, y ; t)$ is the fundamental solution of the Dirichlet problem

$$
\begin{cases}U_{t}=\varepsilon \Delta U & \text { in } \Omega, \\ U=0 & \text { on } \partial \Omega\end{cases}
$$

Since $I_{2}=\int_{0}^{t} \int_{\Omega} e^{u(y, s)} G(x, y ; t-s) d y d s \geq 0$, here we only need to estimate $I_{1}$.

Choose $\delta>0$ such that $B_{a, \delta} \subset \Omega$ and $\varphi(x) \geq \frac{M}{2}$ in $B_{a, \delta}$, and rewrite $I_{1}$ as

$$
I_{1}=\int_{B_{x, \delta}} \varphi(y) G(x, y ; t) d t+\int_{\Omega \backslash B_{x, \delta}} \varphi(y) G(x, y ; t) d y .
$$

Then by Lemma 2.1 of [6] we have that there exist $c_{0}, \alpha, \varepsilon_{0}>0$ with $\alpha<\delta / 2$ such that if $0<\varepsilon \leq \varepsilon_{0}$, then

$$
G(x, y, z) \geq\left(1-e^{-c_{0} / \varepsilon}\right) \frac{1}{(4 \pi \varepsilon t)^{N / 2}} e^{-\frac{|x-y|^{2}}{4 \varepsilon t}}
$$

for $(x, y, t) \in B_{a, \alpha} \times B_{x, \alpha} \times(0, \tau)$. Hence, for $(x, t) \in B_{a, \alpha} \times(0, \tau)$,

$$
\int_{B_{x, \alpha}} \varphi(y) G(x, y, t) d y \geq \frac{M C}{2}\left(1-e^{-c_{0} / \varepsilon}\right)
$$

where $C=\int_{B_{x, \alpha}} \frac{1}{(4 \pi \varepsilon t)^{N / 2}} e^{-\frac{|x-y|^{2}}{4 \varepsilon t}} d y$, and $0<C \leq 1$.

On the other hand, by the comparison principle we have that $0<G(x, y ; t)<\frac{1}{(4 \pi \varepsilon t)^{N / 2}} \times$ $e^{-\frac{|x-y|^{2}}{4 \varepsilon t}}$. So we derive

$$
\begin{aligned}
\int_{\Omega \backslash B_{x, \alpha}} G(x, y ; t) d y & <\frac{1}{(4 \pi \varepsilon t)^{N / 2}} \int_{\Omega \backslash B_{x, \alpha}} e^{-\frac{|x-y|^{2}}{4 \varepsilon t}} d y \\
& <e^{-\frac{\alpha^{2}}{8 \varepsilon t}} \frac{1}{(4 \pi \varepsilon t)^{N / 2}} \int_{\mathbb{R}^{N}} e^{-\frac{|x-y|^{2}}{8 \varepsilon t}} d y \\
& =2^{N / 2} e^{-\frac{\alpha^{2}}{8 \varepsilon t}} .
\end{aligned}
$$

Thus, $\int_{\Omega \backslash B_{x, \alpha}} \varphi(y) G(x, y ; t) d y \geq-m \int_{\Omega \backslash B_{x, \alpha}} G(x, y ; t) d y \geq-2^{N / 2} m e^{-\frac{\alpha^{2}}{8 \varepsilon t}}$ and

$$
I_{1} \geq \frac{M C}{2}\left(1-e^{-c_{0} / \varepsilon}\right)-2^{N / 2} m e^{-\frac{\alpha^{2}}{8 \varepsilon t}}, \quad x \in B_{a, \alpha}
$$

for $t \in(0, \min \{\tau, T(\varepsilon)\})$.

Consequently, the solution $u$ of (1.1) is estimated as

$$
u(x, t) \geq \frac{M C}{2}\left(1-e^{-c_{0} / \varepsilon}\right)-2^{N / 2} m e^{-\frac{\alpha^{2}}{8 \varepsilon t}}, \quad x \in B_{a, \alpha}
$$

for $t \in(0, \min \{\tau, T(\varepsilon)\})$. If $\varepsilon>0$ is small enough, it is easy to see that the right-hand side of (2.2) is positive. The proof of Lemma 2.1 is complete. 


\section{Proof of Theorem 1.1}

Proof of Theorem 1.1 By the comparison principle we have

$$
0<T_{M}<T_{m} \quad \text { and } \quad T(\varepsilon) \geq T_{M}=e^{-M} .
$$

It is sufficient to show that

$$
\limsup _{\varepsilon \rightarrow 0} T(\varepsilon) \leq T_{M}
$$

For convenience, we assume that the origin is contained in $\Omega$ and $\varphi(0)=M$. Fix $\tau \in$ $\left(T_{M}, T_{m}\right)$. By Lemma 2.1 we have that there exists a ball $D \subset \Omega$ centered at the origin and $\varepsilon_{1}>0$ such that if $0<\varepsilon \leq \varepsilon_{1}$, then $u(x, t)>0$ in $D$ for $t \in(0, \min \{\tau, T(\varepsilon)\})$. For every $\mu>0$, there exists $\delta_{\mu}>0$ small enough such that $B_{\mu}=\left\{x \in \mathbb{R}^{N}|| x \mid \leq \delta_{\mu}\right\} \subset D$ and $M-\mu \leq \varphi(x) \leq$ $M$ for $x \in B_{\mu}$. We choose a continuous and radially symmetric function $\widetilde{\varphi}(x)$ on $\bar{D}$ that is decreasing in $|x|$ and satisfies

$$
0 \leq \widetilde{\varphi}(x) \leq \varphi(x), \quad x \in D,
$$

and

$$
\widetilde{\varphi}(x) \equiv M-\mu, \quad x \in B_{\mu} .
$$

Assume that $\tilde{u}$ is a solution of

$$
\begin{cases}\tilde{u}_{t}=\varepsilon \Delta \tilde{u}+e^{\tilde{u}} & \text { in } D \times(0,+\infty) \\ \tilde{u}(x, t)=0 & \text { on } \partial D \times(0,+\infty) \\ \tilde{u}(x, 0)=\tilde{\varphi}(x) & \text { in } D\end{cases}
$$

Then by comparison we have

$$
u(x, t) \geq \tilde{u}(x, t) \quad \text { in } D
$$

for $t \in(0, \min \{\tau, T(\varepsilon)\})$.

Assume that $\widetilde{U}$ is a solution of

$$
\begin{cases}\widetilde{U}_{t}=\varepsilon \Delta \widetilde{U} & \text { in } D \times(0,+\infty), \\ \widetilde{U}(x, t)=0 & \text { on } \partial D \times(0,+\infty), \\ \widetilde{U}(x, 0)=\widetilde{\varphi}(x) & \text { in } D,\end{cases}
$$

and we define

$$
\underline{u}(x, t)=z(t ; \tilde{U}(x, t))=-\ln \left[e^{-\widetilde{U}(x, t)}-t\right] .
$$

Denote the blowup time of $\underline{u}$ by $\bar{T}(\varepsilon)$, that is,

$$
\bar{T}(\varepsilon)=\sup \left\{t>0 \mid e^{-\|\widetilde{U}(\cdot, t)\|_{\infty}}>s, \forall s \in(0, t)\right\} .
$$


Obviously,

$$
\underline{u}(x, t)=-\ln \left[e^{-\widetilde{U}(x, t)}-t\right] \geq-\ln \left[e^{-\widetilde{U}(x, t)}\right]=\widetilde{U}(x, t) \geq 0,
$$

and since

$$
z_{\theta}=e^{-\theta} \cdot e^{z}, \quad z_{\theta \theta}=t e^{-\theta} \cdot e^{2 z}, \quad z_{t}=e^{z},
$$

we derive

$$
\begin{aligned}
\underline{u}_{t}(x, t)-\varepsilon \Delta \underline{u}(x, t)-e^{\underline{u}}= & z_{t}(t ; \widetilde{U})+z_{\theta}(t ; \widetilde{U}) \widetilde{U}_{t}-z_{\theta \theta}(t ; \widetilde{U})|\nabla \widetilde{U}|^{2} \\
& -\varepsilon z_{\theta}(t ; \widetilde{U}) \Delta \widetilde{U}-e^{z(t ; \widetilde{U})} \\
= & -t e^{-\theta} e^{2 z} \\
\leq & 0 .
\end{aligned}
$$

Therefore, we see that $\underline{u}(x, t)$ is a subsolution of (3.2), and by comparison we get

$$
0 \leq \underline{u}(x, t) \leq \widetilde{u}(x, t) \leq u(x, t)
$$

for $t \in(0, \min \{\tau, T(\varepsilon)\})$ and $x \in D$, and we can derive that $T(\varepsilon) \leq \bar{T}(\varepsilon)$. So, it is sufficient to show that

$$
\limsup _{\varepsilon \rightarrow 0} \bar{T}(\varepsilon) \leq T_{M}
$$

Now we estimate $\|\widetilde{U}(\cdot, t)\|_{\infty}$. By the choice of $\widetilde{\varphi}$ we know that it must attain at the origin. Suppose that $\widetilde{G}$ is the fundamental solution of $\widetilde{U}_{t}=\varepsilon \Delta \widetilde{U}$ in $D$ with the homogeneous Dirichlet condition and that $\varepsilon_{1}$ and $\delta_{\mu}$ are positive and small enough. Then, by Lemma 2.1 of [6] on $D$ it is easy to find that there is a constant $\tilde{c}_{0}>0$ such that, for $0<\varepsilon \leq \varepsilon_{1}$,

$$
\widetilde{G}(0, y ; t) \geq\left(1-e^{-\tilde{c}_{0} / \varepsilon}\right) \frac{1}{(4 \pi \varepsilon t)^{N / 2}} e^{-\frac{|y|^{2}}{4 \varepsilon t}}
$$

for $(y, t) \in B_{\mu} \times(0, \tau)$. Hence, since $M-\mu \leq \varphi \leq M$ for $x \in B_{\mu}$, we obtain

$$
\begin{aligned}
\|\widetilde{U}(\cdot, t)\|_{\infty} & =\widetilde{U}(0, t) \\
& =\int_{B_{\mu}} \tilde{\varphi}(y) \widetilde{G}(0, y ; t) d y+\int_{D \backslash B_{\mu}} \widetilde{\varphi}(y) \widetilde{G}(0, y ; t) d y \\
& \geq(M-\mu)\left(1-e^{-\tilde{c}_{0} / \varepsilon}\right) \frac{1}{(4 \pi \varepsilon t)^{N / 2}} \int_{B_{\mu}} e^{-\frac{|y|^{2}}{4 \varepsilon t}} d y \\
& =(M-\mu)\left(1-e^{-\tilde{c}_{0} / \varepsilon}\right)\left[1-\frac{1}{(4 \pi \varepsilon t)^{N / 2}} \int_{\mathbb{R}^{N} \backslash B_{\mu}} e^{-\frac{|y|^{2}}{4 \varepsilon t}} d y\right] \\
& \geq(M-\mu)\left(1-e^{-\tilde{c}_{0} / \varepsilon}\right)\left(1-2^{N / 2} e^{-\frac{\delta_{\mu}^{2}}{8 \varepsilon t}}\right)
\end{aligned}
$$

for $0<t<\tau$. 
By the last estimate, for every $\mu>0$ and for $\varepsilon>0$ small enough, we have

$$
\|\widetilde{U}(\cdot, t)\|_{\infty} \geq M-2 \mu>0
$$

for $t \in(0, \tau)$. Then

$$
e^{-\|\widetilde{U}(\cdot, t)\|_{\infty}} \leq e^{-M} \cdot e^{2 \mu}=T_{M} \cdot e^{2 \mu}
$$

for $t \in(0, \tau)$. Since $T_{M}<T_{M} \cdot e^{2 \mu}<\tau$ for small $\mu$, we have

$$
e^{-\left\|\widetilde{U}\left(\cdot, T_{M} \cdot e^{2 \mu}\right)\right\|_{\infty}} \leq T_{M} \cdot e^{2 \mu} .
$$

By the definition of $\bar{T}(\varepsilon)$ we obtain

$$
\bar{T}(\varepsilon) \leq T_{M} \cdot e^{2 \mu}
$$

Since $\mu>0$ is arbitrary, (3.4) holds. The proof of Theorem 1.1 is complete.

\section{Proof of Theorem 1.2}

In the following, we derive the lower and upper bounds of $T(\varepsilon)$ and give the proof of Theorem 1.2. First, we prove an upper estimate.

Proposition 4.1 If $\varphi$ satisfies (1.4), $\varphi\left(x_{0}\right)=\max _{x \in \bar{\Omega}} \varphi(x)$, and $\varepsilon>0$ is small enough, then we have

$$
T(\varepsilon) \leq e^{-\varphi\left(x_{0}\right)}+e^{-2 \varphi\left(x_{0}\right)}\left|\Delta \varphi\left(x_{0}\right)\right| \varepsilon+\circ(\varepsilon) .
$$

Proof Let $w(x, t)=-\ln (V(x, t)-t)$, where $V(x, t)=U(x, \varepsilon t)$, and $U(x, \tau)$ satisfies $U_{\tau}=\Delta U$. Then

$$
\begin{aligned}
w_{t}-\varepsilon \Delta w-e^{w} & =-\frac{V_{t}-1}{V-t}+\varepsilon \frac{\Delta V}{V-t}-\varepsilon \frac{|\nabla V|^{2}}{(V-t)^{2}}-\frac{1}{V-t} \\
& \leq-\frac{V_{t}-\varepsilon \Delta V}{V-t}=0 .
\end{aligned}
$$

So we see that $w$ is a subsolution.

Next, let $U(x, \tau)$ satisfy

$$
\begin{aligned}
& U(x, 0)=e^{-\varphi(x)}, \quad x \in \Omega, \\
& U(x, \tau)=\frac{\tau}{\varepsilon}+1, \quad(x, t) \in \partial \Omega \times(0, C \varepsilon),
\end{aligned}
$$

where $C>0$ is a constant. Then

$$
\begin{aligned}
& w(x, 0)=-\ln (U(x, 0))=\varphi(x)=u(x, 0), \quad x \in \Omega, \\
& w(x, t)=-\ln (U(x, \varepsilon t)-t)=0, \quad(x, t) \in \partial \Omega \times(0, C) .
\end{aligned}
$$


Thus, by the comparison principle

$$
u(x, t) \geq w(x, t), \quad(x, t) \in \Omega \times(0, C) .
$$

Notice that the boundary values of $U(x, \tau)$ are bounded, that is, $0 \leq U(x, \tau) \leq C+1$ for $(x, \tau) \in \partial \Omega \times(0, C \varepsilon)$, so we can write

$$
U\left(x_{0}, \varepsilon t\right)=U\left(x_{0}, 0\right)+\varepsilon t U_{\tau}\left(x_{0}, 0\right)+\circ(\varepsilon)
$$

for $0<t<C$.

We take $\tilde{t}=U\left(x_{0}, \varepsilon \tilde{t}\right)$. By the definition of $w(x, t), w(x, t) \rightarrow \infty$ as $t \rightarrow \tilde{t}$, so that the function $w(x, t)$ blows up at time $\leq \tilde{t}$.

Choosing $C>U\left(x_{0}, 0\right)+1$, by $(4.1)$ we have

$$
\begin{aligned}
\tilde{t} & =U\left(x_{0}, \varepsilon \tilde{t}\right) \\
& =U\left(x_{0}, 0\right)+\varepsilon \tilde{t} U_{\tau}\left(x_{0}, 0\right)+o(\varepsilon) \\
& =U\left(x_{0}, 0\right)+\varepsilon U\left(x_{0}, \varepsilon \tilde{t}\right) U_{\tau}\left(x_{0}, 0\right)+o(\varepsilon) \\
& =U\left(x_{0}, 0\right)+\varepsilon\left[U\left(x_{0}, 0\right)+\varepsilon \tilde{t} U_{\tau}\left(x_{0}, 0\right)+o(\varepsilon)\right] U_{\tau}\left(x_{0}, 0\right)+o(\varepsilon) \\
& =U\left(x_{0}, 0\right)+\varepsilon U\left(x_{0}, 0\right) \Delta U+o(\varepsilon) .
\end{aligned}
$$

Since $U\left(x_{0}, 0\right)=e^{-\varphi\left(x_{0}\right)}$ and $\nabla \varphi\left(x_{0}\right)=0$, we have $\Delta U\left(x_{0}, 0\right)=-e^{-\varphi\left(x_{0}\right)} \Delta \varphi\left(x_{0}\right)$. Thus, as $\varepsilon \rightarrow 0$,

$$
\tilde{t}=e^{-\varphi\left(x_{0}\right)}+e^{-2 \varphi\left(x_{0}\right)}\left|\Delta \varphi\left(x_{0}\right)\right| \varepsilon+\circ(\varepsilon) .
$$

Since $T(\varepsilon) \leq \tilde{t}$, Proposition 4.1 is proved.

Now, we show a lower bound of $T(\varepsilon)$.

Proposition 4.2 If $\varphi$ satisfies (1.4), $\varphi\left(x_{0}\right)=\max _{x \in \bar{\Omega}} \varphi(x), \Delta \varphi\left(x_{0}\right)<0$, and $\varepsilon>0$ is small enough, then we have

$$
T(\varepsilon) \geq e^{-\varphi\left(x_{0}\right)}+\frac{1}{4} e^{-2 \varphi\left(x_{0}\right)}\left|\Delta \varphi\left(x_{0}\right)\right| \varepsilon+\circ(\varepsilon)
$$

Proof Without loss of generality, let $x_{0}=0$. We construct a supersolution of the form

$$
w(x, t)=-\ln \left(e^{-\varphi(0)}-t\right)+W(x, t), \quad(x, t) \in \Omega \times(0, C),
$$

where $C \in\left(0, e^{-\varphi(0)}\right)$ is a constant, $W(x, t)=Z(x, \varepsilon t)$, and $Z(x, \tau)$ satisfies

$$
\begin{cases}Z_{\tau}=\Delta Z, & x \in \Omega, 0<\tau<C \varepsilon, \\ Z(x, 0)=\varphi(x)-\varphi(0), & x \in \Omega, \\ Z(x, \tau)=-\varphi(0), & x \in \partial \Omega, 0<\tau<C \varepsilon .\end{cases}
$$


Clearly, by the maximum principle, $Z \leq 0$, and hence $W(x, t)=Z(x, \varepsilon t) \leq 0$ for $(x, t) \in$ $\Omega \times(0, C)$. We get

$$
\begin{aligned}
w_{t}-\varepsilon \Delta w-e^{w} & =\frac{1}{e^{\varphi(0)}-t}+W_{t}-\varepsilon \Delta W-\frac{e^{W}}{e^{-\varphi(0)}-t} \\
& =\frac{1-e^{W}}{e^{-\varphi(0)}-t} \geq 0
\end{aligned}
$$

and also

$$
w(x, 0)=\varphi(x)=u(x, 0), \quad x \in \Omega,
$$

and

$$
w(x, t)=-\ln \left(e^{\varphi(0)}-t\right)-\varphi(0)>0, \quad x \in \partial \Omega, 0<t<C .
$$

It follows that

$$
w(x, t) \geq u(x, t), \quad x \in \partial \Omega, 0<t<C .
$$

Choose $\mu>0$ small enough and $\eta>0$ sufficiently small depending on $\mu$ such that

$$
-\Delta \varphi(x)>-\Delta \varphi(0)-\mu \quad \text { for }|x|<\eta .
$$

Then, when $|x|<\eta$,

$$
\begin{aligned}
Z(x, \tau) & =Z(x, 0)+Z_{\tau}(x, 0) \tau+o(\tau) \\
& =\varphi(x)-\varphi(0)+\Delta \varphi(x) \tau+o(\tau) \\
& \leq \Delta \varphi(x) \tau+o(\tau) \\
& \leq(\Delta \varphi(0)+\mu) \tau+o(\tau)
\end{aligned}
$$

as $\tau \rightarrow 0$. When $|x| \geq \eta$ and $x \in \Omega, Z(x, \tau) \leq 0$. It follows that, when $|x|<\eta$,

$$
\begin{aligned}
u(x, C) & \leq w(x, C)=-\ln \left(e^{-\varphi(0)}-C\right)+W(x, C) \\
& =-\ln \left(e^{-\varphi(0)}-C\right)+(\Delta \varphi(0)+\mu) \varepsilon C+o(\varepsilon)
\end{aligned}
$$

as $\varepsilon \rightarrow 0$, and, when $|x| \geq \eta$,

$$
\begin{aligned}
u(x, C) & \leq w(x, C)=-\ln \left(e^{-\varphi(0)}-C\right)+W(x, C) \\
& \leq-\ln \left(e^{-\varphi(0)}-C\right) .
\end{aligned}
$$

Denote by $\tilde{u}(x, t)$ the solution of

$$
\begin{cases}\tilde{u}_{t}=\varepsilon \Delta \tilde{u}+e^{\tilde{u}}, & (x, t) \in \Omega \times(C,+\infty), \\ \tilde{u}(x, t)=0, & (x, t) \in \partial \Omega \times(C,+\infty), \\ \tilde{u}(x, C)=w(x, C), & x \in \Omega,\end{cases}
$$


and by $\widetilde{T}$ the blowup time for $\tilde{u}$. By the comparison principle,

$$
T(\varepsilon) \geq \widetilde{T} \geq C+e^{-\max _{\Omega} u(\cdot, C)} .
$$

On the one hand, when $\max _{\Omega} u(\cdot, C)$ arrives at $\{x|| x \mid<\eta\}$, we have

$$
\begin{aligned}
T(\varepsilon) & \geq C+e^{-\max _{\Omega} u(\cdot, C)} \\
& \geq C+e^{\left[\ln \left(e^{-\varphi(0)}-C\right)-(\Delta \varphi(0)+\mu) \varepsilon C+o(\varepsilon)\right]} \\
& \geq C+\left(e^{-\varphi(0)}-C\right)[1-(\Delta \varphi(0)+\mu) \varepsilon C+o(\varepsilon)] \\
& \geq e^{-\varphi(0)}-\varepsilon C\left(e^{-\varphi(0)}-C\right)[\Delta \varphi(0)+\mu]+o(\varepsilon) .
\end{aligned}
$$

Since $\mu>0$ is arbitrary and $\Delta \varphi(0)<0$, we get

$$
T(\varepsilon) \geq e^{-\varphi(0)}+\varepsilon C\left(e^{-\varphi(0)}-C\right)|\Delta \varphi(0)|+\circ(\varepsilon) .
$$

In order to get the optimal estimate, we choose $C=\frac{1}{2} e^{-\varphi(0)}$, which maximizes the coefficient of $\varepsilon$, that is,

$$
T(\varepsilon) \geq e^{-\varphi\left(x_{0}\right)}+\frac{1}{4} e^{-2 \varphi\left(x_{0}\right)}\left|\Delta \varphi\left(x_{0}\right)\right| \varepsilon+o(\varepsilon) .
$$

Since $\max _{\Omega} u(\cdot, C)$ arrives at $\{x|| x \mid \geq \eta\}$, we have

$$
\begin{aligned}
T(\varepsilon) & \geq C+e^{-\max _{\Omega} u(\cdot, C)} \\
& \geq C+e^{\ln \left(e^{-\varphi(0)}-C\right)} \\
& =e^{-\varphi(0)} .
\end{aligned}
$$

In conclusion,

$$
T(\varepsilon) \geq e^{-\varphi(0)}+\frac{1}{4} e^{-2 \varphi(0)}|\Delta \varphi(0)| \varepsilon+o(\varepsilon) .
$$

Proposition 4.2 is proved.

Combining Propositions 4.1 and 4.2, we get the proof of Theorem 1.2.

\section{Proof of Theorem 1.3}

In the following, we derive the lower and upper bounds of $T(\varepsilon)$ and give a proof of Theorem 1.3. We first prove an upper bound of $T(\varepsilon)$.

Proposition 5.1 If $\varphi$ satisfies (1.4), $\varphi\left(x_{0}\right)=\max _{x \in \bar{\Omega}} \varphi(x)$, and, moreover, there exist constants $c_{0}, k>0, k \neq 1$ such that $\varphi(x) \geq \varphi\left(x_{0}\right)-c_{0}\left|x-x_{0}\right|^{2 k}$ as $x \rightarrow x_{0}$, then, for $\varepsilon>0$ small enough,

$$
T(\varepsilon) \leq e^{-\varphi\left(x_{0}\right)}+c_{0} \gamma_{k} e^{-(k+1) \varphi\left(x_{0}\right)} \varepsilon^{k}+\circ\left(\varepsilon^{k}\right),
$$


where $\gamma_{k}$ satisfies

$$
\int_{\Omega} \frac{e^{-\frac{\left|y-x_{0}\right|^{2}}{4 \tau}}}{(4 \pi \tau)^{N / 2}}\left|y-x_{0}\right|^{2 k} d y=\gamma_{k} \tau^{k}+o\left(\tau^{k}\right) \quad \text { as } \tau \rightarrow 0 .
$$

Proof Similarly to the proof of Proposition 4.1, we construct a subsolution as

$$
w(x, t)=-\ln (V(x, t)-t),
$$

where $V(x, t)=U(x, \varepsilon t)$, and $U(x, \tau)$ satisfies $U_{\tau}=\Delta U$. Then by the proof of Proposition 4.1 we get

$$
u(x, t) \geq w(x, t)
$$

for $0<t<C$. Assuming that $\mu>0$, we take $\delta_{\mu}>0$ small enough such that $B_{\mu}=\left\{x \in \mathbb{R}^{N} \| x-\right.$ $\left.x_{0} \mid<\delta_{\mu}\right\} \subset \Omega$ and $\varphi(x) \geq \varphi\left(x_{0}\right)-c_{0}\left|x-x_{0}\right|^{2 k}$ for $x \in B_{\mu}$.

Next, we consider the Dirichlet problem

$$
\begin{cases}U_{t}=\Delta U & \text { in } B_{\mu} \\ U(x, t)=0 & \text { on } \partial B_{\mu}\end{cases}
$$

and we denote its fundamental solution by $G(x, y ; t)$. Then by a comparison to the fundamental solution of $U_{t}=\Delta U$ on $\mathbb{R}^{N}$ we derive

$$
G(x, y ; t) \leq \frac{e^{-\frac{|x-y|^{2}}{4 t}}}{(4 \pi t)^{N / 2}} \quad \text { for }(x, y ; t) \in B_{\mu} \times B_{\mu} \times(0, \infty) .
$$

Thus,

$$
\begin{aligned}
U\left(x_{0}, \tau\right)= & \int_{B_{\mu}} e^{-\varphi(y)} \frac{e^{-\frac{\left|x_{0}-y\right|^{2}}{4 \tau}}}{(4 \pi \tau)^{N / 2}} d y \\
\leq & \int_{B_{\mu}}\left[e^{-\varphi\left(x_{0}\right)}+e^{-\varphi\left(x_{0}\right)} c_{0}\left|y-x_{0}\right|^{2 k}+\circ\left(\left|y-x_{0}\right|^{2 k}\right)\right] \frac{e^{-\frac{\left|x_{0}-y\right|^{2}}{4 \tau}}}{(4 \pi \tau)^{N / 2}} d y \\
\leq & \int_{\mathbb{R}^{N}}\left[e^{-\varphi\left(x_{0}\right)}+\circ\left(\left|y-x_{0}\right|^{2 k}\right)\right] \frac{e^{-\frac{\left|x_{0}-y\right|^{2}}{4 \tau}}}{(4 \pi \tau)^{N / 2}} d y \\
& +\int_{B_{\mu}}\left[e^{-\varphi\left(x_{0}\right)} c_{0}\left|y-x_{0}\right|^{2 k}\right] \frac{e^{-\frac{\left|x_{0}-y\right|^{2}}{4 \tau}}}{(4 \pi \tau)^{N / 2}} d y \\
\leq & e^{-\varphi\left(x_{0}\right)}+\circ\left(\delta_{\mu}^{2 k}\right)+e^{-\varphi\left(x_{0}\right)} c_{0} \gamma_{k} \tau^{k}+\circ\left(\tau^{k}\right) .
\end{aligned}
$$

Since $\delta_{\mu}>0$ is sufficiently small,

$$
U\left(x_{0}, \tau\right) \leq e^{-\varphi\left(x_{0}\right)}+e^{-\varphi\left(x_{0}\right)} c_{0} \gamma_{k} \tau^{k}+\circ\left(\tau^{k}\right) .
$$


We take $\tilde{t}=U\left(x_{0}, \varepsilon \tilde{t}\right)$. By the definition of $w(x, t)$ we know that $w(x, t) \rightarrow \infty$ as $t \rightarrow \tilde{t}$. So the function $w(x, t)$ blows up at time $\leq \tilde{t}$. We have

$$
\begin{aligned}
\tilde{t} & =U\left(x_{0}, \varepsilon \tilde{t}\right) \leq e^{-\varphi\left(x_{0}\right)}+e^{-\varphi\left(x_{0}\right)} c_{0} \gamma_{k}(\varepsilon \tilde{t})^{k}+\circ\left(\varepsilon^{k}\right) \\
& \leq e^{-\varphi\left(x_{0}\right)}+e^{-\varphi\left(x_{0}\right)} c_{0} \gamma_{k} \varepsilon^{k}\left[U\left(x_{0}, 0\right)+\varepsilon \tilde{t} U_{\tau}\left(x_{0}, 0\right)+o(\varepsilon)\right]^{k}+\circ\left(\varepsilon^{k}\right) \\
& =e^{-\varphi\left(x_{0}\right)}+e^{-\varphi\left(x_{0}\right)} c_{0} \gamma_{k} \varepsilon^{k} U\left(x_{0}, 0\right)^{k}+\circ\left(\varepsilon^{k}\right) \\
& =e^{-\varphi\left(x_{0}\right)}+c_{0} \gamma_{k} e^{-(k+1) \varphi\left(x_{0}\right)} \varepsilon^{k}+\circ\left(\varepsilon^{k}\right) .
\end{aligned}
$$

Since $T(\varepsilon) \leq \tilde{t}$, we complete the proof of Proposition 5.1 .

We next give a lower estimate of $T(\varepsilon)$.

Proposition 5.2 If $\varphi$ satisfies (1.4), $\varphi\left(x_{0}\right)=\max _{x \in \bar{\Omega}} \varphi(x)$, and, moreover, there exist constants $c_{0}, k>0, k \neq 1$ such that $\varphi(x) \leq \varphi\left(x_{0}\right)-c_{0}\left|x-x_{0}\right|^{2 k}$ as $x \rightarrow x_{0}$, then,for $\varepsilon>0$ small enough,

$$
T(\varepsilon) \geq e^{-\varphi\left(x_{0}\right)}+c_{0} \zeta_{k} \gamma_{k} e^{-(k+1) \varphi\left(x_{0}\right)} \varepsilon^{k}+\circ\left(\varepsilon^{k}\right)
$$

where $\zeta_{k}=k^{k} /(k+1)^{k+1}$, and $\gamma_{k}$ satisfies

$$
\int_{\Omega} \frac{e^{-\frac{\left|y-x_{0}\right|^{2}}{4 \tau}}}{(4 \pi \tau)^{N / 2}}\left|y-x_{0}\right|^{2 k} d y=\gamma_{k} \tau^{k}+\circ\left(\tau^{k}\right) \quad \text { as } \tau \rightarrow 0
$$

Proof Without loss of generality, taking $x_{0}=0$, we construct a supersolution as

$$
w(x, t)=-\ln \left(e^{-\varphi(0)-t}\right)+W(x, t)
$$

for $0<t<C$, where $C \in\left(0, e^{-\varphi(0)}\right), w(x, t)=Z(x, \varepsilon t)$, and $Z(x, \tau)$ satisfies equation (4.2).

Then we estimate $Z(x, \tau)$. There exists $\eta>0$ such that $B_{\eta}=\left\{x \in \mathbb{R}^{N}|| x \mid<\eta\right\} \subset \Omega$ and $\varphi(x) \leq \varphi(0)-c_{0}|x|^{2 k}$ for $x \in B_{\eta}$.

By the fundamental solution we write $Z(x, \tau)$ as

$$
Z(x, \tau)=\int_{B_{\eta}}(\varphi(y)-\varphi(0)) \frac{e^{-\frac{|x-y|^{2}}{4 \tau}}}{(4 \pi \tau)^{N / 2}} d y .
$$

When $|x|<\eta$,

$$
\begin{aligned}
Z(x, \tau) & \leq \int_{B_{\eta}}\left[-c_{0}|y|^{2 \alpha} \frac{e^{-\frac{|x-y|^{2}}{4 \tau}}}{(4 \pi \tau)^{N / 2}}\right] d y \\
& =-c_{0} \gamma_{k} \tau^{k}+o\left(\tau^{k}\right),
\end{aligned}
$$

whereas when $|x| \geq \eta$ and $x \in \Omega, Z(x, \tau) \leq 0$. It follows that, when $|x|<\eta$,

$$
\begin{aligned}
u(x, C) & \leq w(x, C) \\
& =-\ln \left(e^{-\varphi(0)}-C\right)+W(x, C) \\
& =-\ln \left(e^{-\varphi(0)}-C\right)-c_{0} \gamma_{k} \varepsilon^{k} C^{k}+\circ\left(\varepsilon^{k}\right)
\end{aligned}
$$


as $\varepsilon \rightarrow 0$ and that

$$
\begin{aligned}
u(x, C) & \leq w(x, C) \\
& =-\ln \left(e^{-\varphi(0)}-C\right)+W(x, C) \\
& =-\ln \left(e^{-\varphi(0)}-C\right)
\end{aligned}
$$

when $|x| \geq \eta$ and $x \in \Omega$.

By the same arguments as in Proposition 4.2,

$$
T(\varepsilon) \geq C+e^{-\max _{\Omega} u(\cdot, C)} .
$$

On the one hand, if $\max _{\Omega} u(\cdot, C)$ arrives at $\{x|| x \mid<\eta\}$, we have

$$
\begin{aligned}
T(\varepsilon) & \geq C+e^{\left[\ln \left(e^{-\varphi(0)}-c\right)+c_{0} \gamma_{k} \varepsilon^{k} C^{k}+o\left(\varepsilon^{k}\right)\right]} \\
& =e^{-\varphi(0)}+c_{0} \gamma_{k} \varepsilon^{k} C^{k}\left[e^{-\varphi(0)}-c\right]+\circ\left(\varepsilon^{k}\right) .
\end{aligned}
$$

In order to get the optimal estimate, we choose $C=k /(k+1) e^{-\varphi(0)}$, which maximizes the coefficient of $\varepsilon^{k}$, that is,

$$
T(\varepsilon) \geq e^{-\varphi\left(x_{0}\right)}+c_{0} \frac{k^{k}}{(k+1)^{k+1}} \gamma_{k} e^{-(k+1) \varphi\left(x_{0}\right)} \varepsilon^{k}+o\left(\varepsilon^{k}\right)
$$

whereas if $\max _{\Omega} u(\cdot, C)$ arrives at $\{x|| x \mid \geq \eta\}$, then we have

$$
T(\varepsilon) \geq C+e^{-\max _{\Omega} u(\cdot, C)}=e^{-\varphi(0)} .
$$

In conclusion, as $\varepsilon \rightarrow 0$,

$$
T(\varepsilon) \geq e^{-\varphi(0)}+c_{0} \zeta_{k} \gamma_{k} e^{-(k+1) \varphi(0)} \varepsilon^{k}+o\left(\varepsilon^{k}\right)
$$

where $\zeta_{k}=k^{k} /(k+1)^{k+1}$, and $\gamma_{k}$ satisfies

$$
\int_{\Omega} \frac{e^{-\frac{|y|^{2}}{4 \tau}}}{(4 \pi \tau)^{N / 2}}|y|^{2 k} d y=\gamma_{k} \tau^{k}+o\left(\tau^{k}\right) \quad \text { as } \tau \rightarrow 0 .
$$

Now, combining Propositions 5.1 and 5.2, we get the proof of Theorem 1.3.

\section{Extension}

We note that the assumption of (1.4) implies that $\varphi$ is nonnegative or sign-changing function. However, when $\varphi$ is negative, it is very interesting whether the blowup phenomenon still occurs, and if it does, how to describe the asymptotic behavior of the blowup time as $\varepsilon \rightarrow 0$.

When $\varphi$ is negative, letting $v(x, t)=-u(x, t)$, we can change equation (1.1) into

$$
\begin{cases}v_{t}=\varepsilon \Delta v-e^{-v}, & (x, t) \in \Omega \times(0,+\infty), \\ v(x, t)=0, & (x, t) \in \partial \Omega \times(0,+\infty), \\ v(x, 0)=-\varphi(x), & x \in \Omega\end{cases}
$$


We also consider the zero diffusion case of ODE. Suppose that $z(t ; \theta)$ is a solution of the equation

$$
\frac{d z}{d t}=-e^{-z}, \quad z(0 ; \theta)=\theta>0
$$

Then $z(t ; \theta)$ is solved as

$$
z(t ; \theta)=\ln \left(e^{\theta}-t\right)
$$

Clearly, $z(t ; \theta) \rightarrow-\infty$ as $t \rightarrow e^{\theta}$. So we suspect that if $\varphi$ were negative, then the blowup time $T(\varepsilon)$ of (1.1) would satisfy

$$
T(\varepsilon) \rightarrow e^{\|\varphi\|_{\infty}} \quad \text { as } \varepsilon \rightarrow 0
$$

In fact, by comparison, $v(x, t) \leq z\left(t ;\|\varphi\|_{\infty}\right)$, and we have $T(\varepsilon) \leq e^{\|\varphi\|_{\infty}}$, that is, $v$ will reach $-\infty(u$ will reach $+\infty)$ faster than $z$. On the other hand, by the similar arguments as in the proof of Theorem 1.1 we can derive that $T(\varepsilon) \geq e^{\|\varphi\|_{\infty}}$ as $\varepsilon \rightarrow 0$. Therefore, (6.3) holds.

However, when $\varphi$ is negative and $\varphi\left(x_{0}\right)=\min _{\bar{\Omega}} \varphi(x)$, we can easily find that $e^{\varphi\left(x_{0}\right)}<1<$ $e^{-\varphi\left(x_{0}\right)}$, and the arguments in the proofs of Theorems 1.2-1.3 will not be valid for this case. In order to get more accurate estimates of $T(\varepsilon)$ for negative initial data, some new way is needed. We leave it to the interested readers.

\section{Competing interests}

The author declares that she has no competing interests.

\section{Acknowledgements}

This work was supported by the National Natural Science Foundation of China (Nos. 11401458, 11371286), the Special Fund of Education Department (No. 2013JK0586), and the Youth Natural Science Grant (No. 2013JQ1015) of Shaanxi Province of China.

Received: 31 May 2016 Accepted: 8 August 2016 Published online: 20 August 2016

\section{References}

1. Bebernes, J, Eberly, D: Mathematical Problems from Combustion Theory. Appl. Math. Sciences, vol. 83. Springer, Berlin (1989)

2. Friedman, A, Lacey, AA: The blow-up time for solutions of nonlinear heat equations with small diffusion. SIAM J. Math Anal. 18, 711-721 (1987)

3. Gui, C, Wang, X: Life span of solutions of the Cauchy problem for a semilinear heat equation. J. Differ. Equ. 115, 166-172 (1995)

4. Lee, TY, Ni, WM: Global existence, large time behavior and life span of solutions of a semilinear parabolic Cauchy problem. Trans. Am. Math. Soc. 333, 365-378 (1992)

5. Sato, S: Life span of solutions with large initial data for a superlinear heat equation. J. Math. Anal. Appl. 343 1061-1074 (2008)

6. Mizoguchi, N, Yanagida, E: Life span of solutions for a semilinear parabolic problem with small diffusion. J. Math. Anal. Appl. 261, 350-368 (2001)

7. Mizoguchi, N, Yanagida, E: Life span of solution with large initial data in a semilinear parabolic equation. Indiana Univ. Math. J. 50, 591-610 (2001)

8. Giga, Y, Kohn, RV: Asymptotically self-similar blow-up of semilinear heat equations. Commun. Pure Appl. Math. 38, 297-319 (1985)

9. Payne, L, Schaefer, P: Lower bounds for blow-up time in parabolic problems under Dirichlet conditions. J. Math. Anal. Appl. 328(2), 1196-1205 (2007)

10. Payne, L, Schaefer, P: Bounds for the blowup time for the heat equation under nonlinear boundary conditions. Proc. R. Soc. Edinb. A 139, 1289-1296 (2009)

11. Li, RF, Zhu, LP, Zhang, ZC: Quenching time for a semilinear heat equation with a nonlinear Neumann boundary condition. J. Partial Differ. Equ. 27(3), 217-228 (2014)

12. Zhang, QY, Jiang, ZX, Zheng, SN: Blow-up time estimate for a degenerate diffusion equation with gradient absorption. Appl. Math. Comput. 251, 331-335 (2015)

13. Zhang, ZC, Hu, B: Gradient blowup rate for a semilinear parabolic equation. Discrete Contin. Dyn. Syst., Ser. A 26, 767-779 (2010) 
14. Zhang, ZC, Hu, B: Rate estimates of gradient blowup for a heat equation with exponential nonlinearity. Nonlinear Anal. TMA 12, 4594-4601 (2010)

15. Zhang, ZC, Li, Y: Blowup and existence of global solutions to nonlinear parabolic equations with degenerate diffusion. Electron. J. Differ. Equ. 2013, 264 (2013)

16. Zhu, LP, Zhang, ZC: Rate of approach to the steady state for a diffusion-convection equation on annular domains. Electron. J. Qual. Theory Differ. Equ. 2012, 39 (2012)

Submit your manuscript to a SpringerOpen ${ }^{\circ}$ journal and benefit from:

- Convenient online submission

- Rigorous peer review

- Immediate publication on acceptance

- Open access: articles freely available online

- High visibility within the field

- Retaining the copyright to your article 